\title{
Pulmonary sequestration and related congenital bronchopulmonary-vascular malformations: nomenclature and classification based on anatomical and embryological considerations
}

\author{
BS CLEMENTS, JO WARNER \\ From the Brompton Hospital, London
}

No satisfactory classification for congenital anomalies of the bronchopulmonary airway and related vasculature has yet been described. An attempt was made to overcome this problem by expanding the concept of "sequestration" (originally intended as a single entity) to include a range of bronchovascular anomalies. This has failed to gain universal acceptance and led us to develop an alternative nomenclature and classification based on simple anatomical description. This paper discusses the background to the new approach while a companion paper applies it to our own cases reviewed during five years.

\section{History and background}

Defining a congenital anatomical lesion of the lung affecting a single structure (for example, bronchus) is relatively simple (for example, bronchial stenosis, bronchogenic cyst). When this lesion includes abnormalities of other major components of lung tissuenamely, arterial supply, venous drainage, and lung parenchyma - differentiation becomes more complex. In 1946 Pryce coined the term sequestration to describe a "disconnected ("dislocated," "ectopic") bronchopulmonary mass or cyst with an anomalous systemic artery supply." "Four of his original seven cases differed considerably from his own definition, which led him to describe three variants of sequestration-namely: abnormal artery to normally connected lung; abnormal artery to both sequestered mass and adjacent normal lung; and abnormal artery

Address for reprint requests: Dr JO Warner, Department of Paediatrics, Brompton Hospital, London SW36HP.

Accepted 11 February 1987 confined to the sequestered mass. ${ }^{2}$ Later the terms intralobar and extralobar were added but they provide no definitive information on the morphology within these lesions. Despite this, many authorities have adhered to Pryce's terminology and consequently encountered problems in defining the variants of sequestration. ${ }^{3-6}$ Many synonyms for the various atypical forms have been proposed, contributing further to the confusion concerning nomenclature $^{37}$ Realisation that these entities may represent variants of a single primary complex of bronchovascular anomalies ${ }^{4689}$ led to the concept of a "sequestration spectrum," a term introduced by Sade in $1974 .{ }^{10}$ This extended term included various combinations of abnormal bronchial connection, arterial supply and venous drainage, so bringing in the scimitar syndrome and its variations. ${ }^{11}$ In 1984 Thilenius again reviewed the sequestration spectrum concept. ${ }^{12}$ He emphasised the wide range of anomalies found by quoting examples of every combination of abnormal bronchial connection, arterial supply, and venous drainage appearing in published reports. He agreed with Sade, and others, ${ }^{13}$ that sequestered areas of lung with normal vasculature should be included in the spectrum. Nevertheless, reports of "atypical" sequestrations continued to inundate published reports, ${ }^{1415}$ indicating that this concept had failed to gain general acceptance. To complicate matters further, associated abnormalities of venous drainage and lung parenchyma were rather neglected in most earlier reports. Recently the importance of defining the venous drainage in these lesions has been emphasised, ${ }^{12} 16$ and our experience further emphasises this aspect (see p 409). Associated abnormalities of lung parenchyma, both within the lesions and in the surrounding lung, have not been widely appreciated, although their existence was noted in Pryce's 


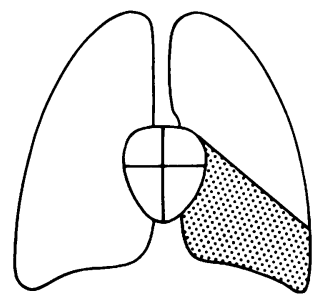

Normal venous drainage

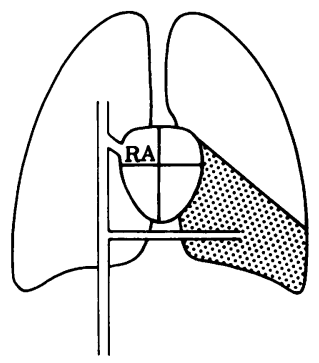

Anomalous venous drainage

Fig 1 Bronchopulmonary malinosculation: an abnormality of the bronchopulmonary airway (hatched area) may affect a localised area (bronchogenic cyst), segment ("sequestration" with normal pulmonary artery supply, cystic adenomatoid malformation), lobe, or lung (agenesis or hypoplasia). There may be associated normal or anomalous venous drainage.

original articles. ${ }^{12}$ We also expand on this feature in our clinical review.

From these descriptions it is apparent that there are essentially four components of these lesions-namely, the tracheobronchial airway, arterial supply, venous drainage, and lung parenchyma. We should therefore be able to construct a definition and classification based on these components that will cover all possible anatomical combinations. In this paper we propose a new nomenclature and classification with supporting evidence from histological and embryological observation. The companion paper ( $p 409$ ) illustrates the clinical advantages of this simple anatomical approach.

\section{NOMENCLATURE AND CLASSIFICATION}

There are several major objections to the use of "sequestration" as a collective term for all

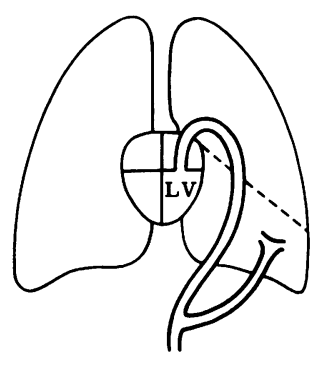

Normal venous drainage

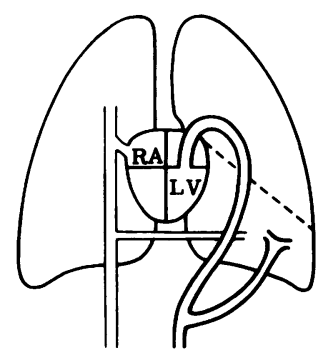

Anomalous venous drainage

Fig 2 Arterial pulmonary malinosculation with a systemic arterial supply to an otherwise normal area of lung. There may be normal or anomalous venous drainage. Evidence of an associated abnormality of the bronchopulmonary tree would alter this to bronchoarterial pulmonary malinosculation (fig 3).



Normal venous drainage

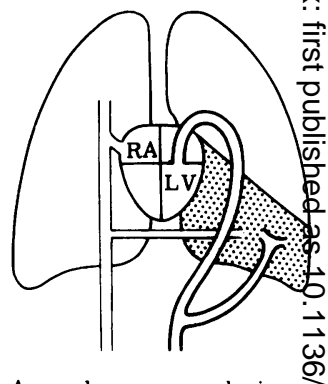

Anomalous venous drainage
Fig 3 Bronchoarterial pulmonary malinosculation, with an abnormal area of lung supplied by an aberrant systemic artery. There may be normal or anomalous venous drainage.

bronchopulmonary-vascular anomalies. These include:

1 The Concise Oxford Dictionary defines seques tration (from the Latin sequestrare, meaning among other things to remove or separate) as to seclude, iso $\infty$ late, set apart. This term is therefore incorrectly applied to the lesions previously described in published reports as, strictly speaking, only "extralobar seques乏 trations" are truly "set apart" from the normal lung 2 The limits of the sequestration spectrum have never been defined; thus the range of anomalie included varies considerably according to the author 3 Most people continue with the impression that "sequestration" is a "disconnected broncho= pulmonary mass or cyst with a systemic arterial supల ply," albeit fully accepting how nebulous this may be Thus there is a natural reluctance to include lesions with normal vasculature in this category.

On the other hand, Stedman's Medical Dictionary (1982) defines malinosculation (Latin: mal $\stackrel{3}{3}$. abnormal; in -in; osculum - mouth) as "the establishô ment of (abnormal) communications by means of small openings or anastomoses, applied especially to the establishment of such communications between already existing blood vessels or other tubular struco tures that come into contact." This definition describes the pathogenesis of these congenital lung lesions exactly. If we are going to classify thent accordingly, we must use this appropriate term. N

Thus pulmonary malinosculation simply describes congenitally abnormal connection or opening of one or more components of the bronchopulmonary vascular complex. The various combinations of these components give rise to a wide spectrum of abnormalio ities. The lesions thus formed can then be classifie $\bar{\Phi}$ simply according to their abnormal anatomical com ponents. The classification is constructed in thre steps. The first step describes the abnormality of bronchopulmonary airway or arterial blood supply of both. The possible combinations of these two comp 
ponents form a spectrum of lesions, as illustrated in figures 1-3. At one end of the spectrum is absence or abnormality of bronchopulmonary airway with normal pulmonary artery blood supply (fig 1 ); this is bronchial pulmonary malinosculation or bronchopulmonary malinosculation. At the other end of the spectrum (fig 2) is an anomalous arterial supply to an area of lung with a normal bronchopulmonary airway; this is arterial pulmonary malinosculation. In between we have all the combinations and gradations of abnormal bronchial and arterial connection. These represent bronchoarterial pulmonary malinosculations (fig 3).

The second step in the classification defines the associated anomalies of venous drainage. The classification is then completed by describing any associated abnormalities of lung parenchyma. Each of these steps will now be reviewed in more detail.

\section{TRACHEOBRONCHOPULMONARY}

\section{MALINOSCULATION}

Disruption of normal airway communication can occur at any level from the trachea down to the alveolus. Disruption may be incomplete (for example, stenosis) or complete (for example, atresia). The lesion may be localised or extend distally to affect a variable segment of the bronchopulmonary tree. These lesions are conveniently divided into those affecting proximal major airways (tracheopulmonary malinosculation), such as tracheal stenosis and tracheal cysts, and those affecting the distal airways down to respiratory bronchioles and alveoli (bronchopulmonary malinosculation). This latter group includes lesions such as bronchial stenosis and bronchogenic cysts, and parenchymal lesions with normal pulmonary artery blood supply, such as congenital lung cysts, cystic adenomatoid malformation, and lobar emphysema. Each of these entities may have associated anomalies of venous drainage and lung parenchyma. ${ }^{12}$

\section{ARTERIAL PULMONARY MALINOSCULATION}

Arterial pulmonary malinosculation refers to an aberrant systemic arterial supply to an area of otherwise normal lung. ${ }^{17}$ Most of these lesions, however, also affect the tracheobronchopulmonary airway to some extent, which will mean that they are classified in the following category.

\section{BRONCHOARTERIAL PULMONARY \\ MALINOSCULATION}

Here there is an abnormality of both the bronchopulmonary airway and the arterial blood supply to an area of lung. The abnormality of bronchopulmonary communication may range from absent connection with a blind ending bronchus (agenesis) or atretic membrane to apparently normal patency with a gradation of abnormality in between.491012 Abnormal branching patterns, reduced numbers of bronchial branches, or generalised hypoplasia may also occur. ${ }^{1218}$ The aberrant arterial blood supply can arise as one or more trunks, usually from the abdominal or thoracic aorta. ${ }^{311} \mathrm{An}$ abnormal area of lung sharing a mixed pulmonary and systemic arterial blood supply has been described (see paper on $\mathrm{p} 409$ ). In addition, part of the surrounding normal lung may be supplied by the anomalous artery. ${ }^{1}$

Entities found in this group include sequestrations, lung cysts with aberrant systemic arterial supply, and congenital cystic bronchiectasis. Again, each of these lesions may have associated anomalies of venous drainage or lung parenchyma, or both (for example, scimitar syndrome). ${ }^{18}$

\section{Abnormalities of venous drainage}

Anomalous venous drainage can occur with any congenital lung malformation whether there is an aberrant systemic arterial supply or not (fig $1-3) .{ }^{12}$ The anomalous veins may be single or multiple, and drain directly to the right atrium, the major draining veins in the thorax or abdomen, or to both the right and the left atrium. ${ }^{16}$ Obstruction to venous return is said to occur in some cases. ${ }^{18}$ In one group of patients, particularly where the lesion is found in the right lung, the anomalous vein or veins drain the major part or the whole of that lung, while the aberrant arterial supply is limited to the right lower zone. This mismatched anomalous venous drainage is a characteristic feature of the scimitar syndrome (fig 4). ${ }^{12} 18$

\section{Abnormalities of lung parenchyma}

Here the site of the lesion is described, together with the changes occurring within the lesion (for example, cystic, adenomatous, emphysematous, or containing

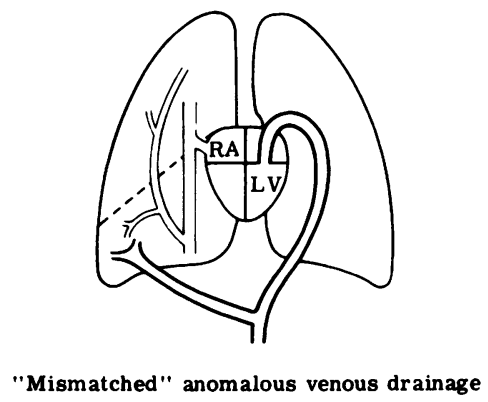

Fig 4 Bronchoarterial pulmonary malinosculation with mismatched anomalous venous drainage - a well recognised variant of the scimitar syndrome. 
Classification of pulmonary malinosculation (first step defining basic abnormality of tracheobronchopulmonary airway connection or arterial blood supply or both, followed by description of associated anomalies of venous drainage and lung parenchyma)

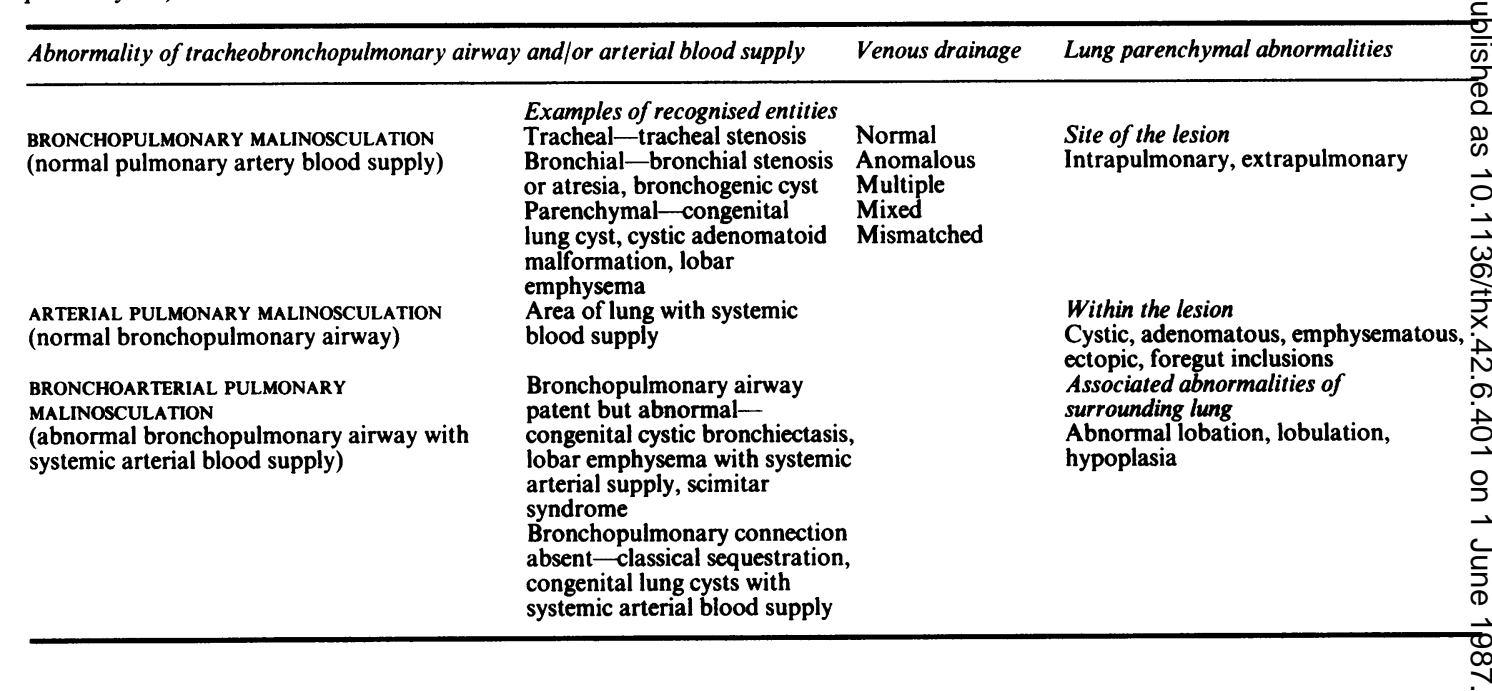

foregut inclusions) and associated abnormalities of surrounding lung parenchyma (for example, hypoplasia and abnormal lobation). The site of the lesion may be intrapulmonary (within the boundary formed by the pleural layer surrounding the lung) or extrapulmonary (ectopic lung tissue lying outside the boundary formed by the pleural layer surrounding the rest of the lung).

The classification of pulmonary malinosculation is summarised in table 1 . The distinct clinical entities within the classification should all retain their common nomenclature. The purpose of this classification is not to replace their accepted terminology but to aid categorisation of these lesions, particularly the more complex varieties. In turn, it is hoped that this will improve the understanding and management of these complicated malformations. A good example of a complex lesion affecting all four major components of lung tissue is a well recognised variant of the scimitar syndrome usually presenting in infancy. ${ }^{1618-20}$ This lesion is situated in the lower half of the right lung with abnormal lobation and bronchial branching, anomalous systemic arterial supply and venous drainage, and associated lung hypoplasia. A characteristic feature is the "mismatch" between the aberrant systemic arterial supply to the right lower zone and the anomalous venous drainage of the whole or major portion of the right lung (fig 4). ${ }^{18-20}$ According to our terminology, this malformation would be classified as bronchoarterial pulmonary malinosculation with mismatched anomalous venous drainage and ipsilateral lung hypoplasia. In a clinical context, each component would then need to be further defined-for example, in terms of the degree of disruption in airway communication and the site of anomalous venous return. The information, with the clinical history and haemodynamic data, is essentiaf for appropriate patient management.

\section{Discussion}

Application of a unifying concept to congenital lung malformations is certainly not new. In 1974 Buntairôे suggested that lobar emphysema, cystic adenomatoid malformation, pulmonary sequestration, and bron chogenic cysts in children were a clinical group. ${ }^{2}$ 㟢 Sade's spectrum ranged from an area of "sequestere 6 lung with normal pulmonary artery supply" to "an. area of normal lung with systemic artery supply, much as we have described. ${ }^{10}$ Heitzman went even further, defining the sequestered lung with normag pulmonary artery supply as including congenital mal formations previously classified elsewhere-such a lobar emphysema, congenital lung cysts, and even cystic adenomatoid malformation. ${ }^{13} \mathrm{He}$ warned that, although the terminology suggests that these are dis 0 crete entities, their morphological characteristic overlap to a considerable degree. Demos removed from a five month old infant a lobe whose tissue, botb grossly and microscopically, showed characteristic of "intra- and extralobular sequestration, bronchiap atresia and cystic adenomatoid malformation." 22 Aso early as 1969 Culiner, when comparing cases of "con genital bronchial cystic disease, sequestration an $\$$ congenital cystic bronchiectasis," suggested that thes three entities were variants of a single primary come plex of bronchovascular anomalies. ${ }^{8}$ It would cer tainly assist our understanding of their pathogenesis 
if they could be regarded in this way.

Fundamental to the subject of all malformations is the embryology of the part concerned. Unfortunately, no generally accepted explanation for the development of these lung anomalies has yet been suggested, despite $\mathbf{4 0}$ propositions in Biancalana's paper $^{23}$ and the six most widely publicised theories. ${ }^{124-28}$ We propose a rational sequence of events in lung development, which would account for all lung anomalies that include malinosculation of one or more components of the bronchopulmonaryvascular complex, starting with a few widely accepted observations concerning embryogenesis, namely:
1 Bronchial branching is completed 16 weeks after conception. ${ }^{29}$

2 Pulmonary artery development is similar to the pattern of bronchial branching but follows slightly later. ${ }^{29}$

3 Alveolar development follows transformation of the terminal bronchioles into respiratory bronchioles and, later, saccules after about the 24th week from conception. ${ }^{29}$

4 In addition to the recognised stages of lung development, there are differing rates of growth occurring in various tissues at different times. ${ }^{30}$

5 In the early stages the tips of the dividing bron-

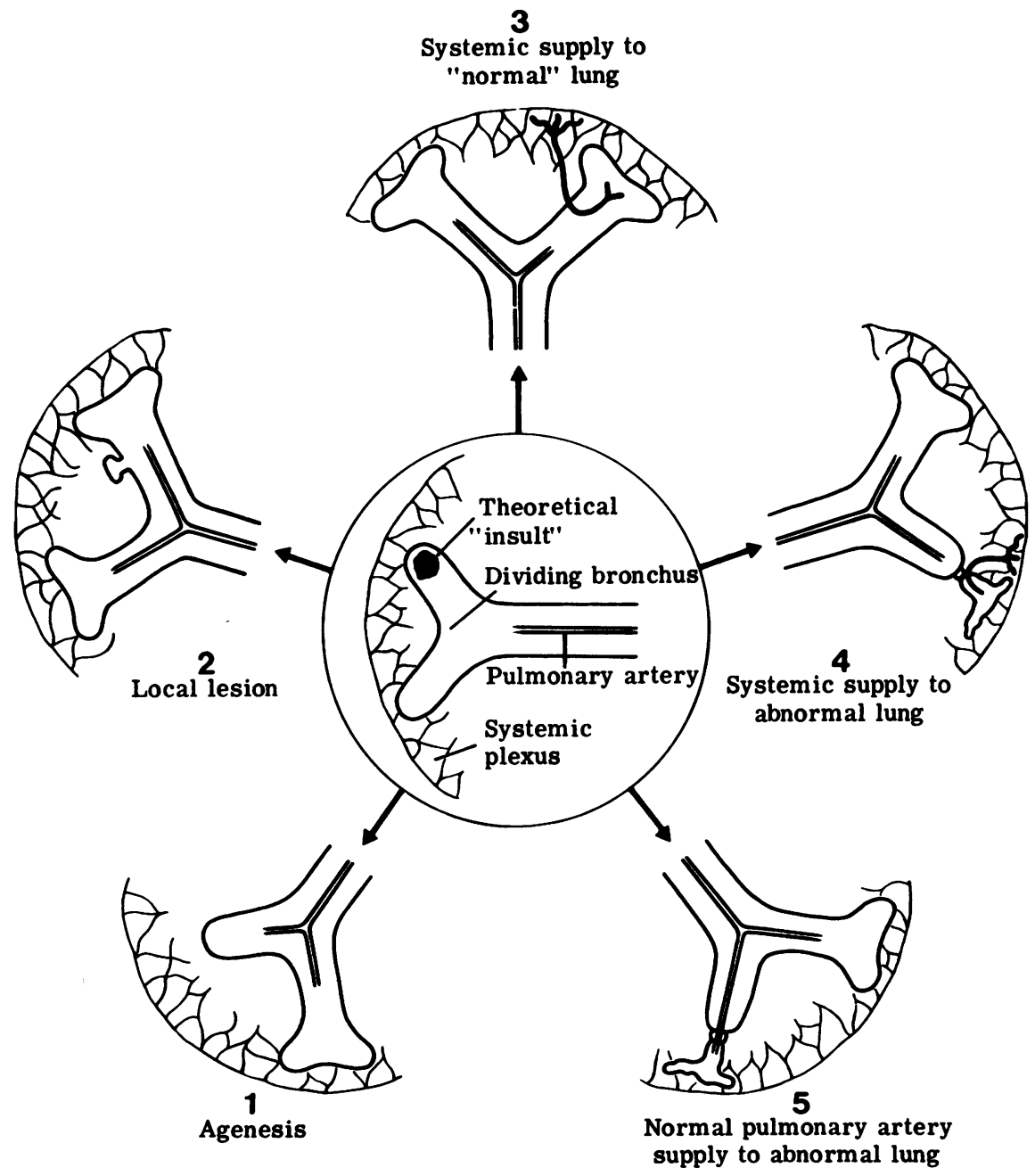

Fig 5 The "wheel" theory of abnormal lung development. After a theoretical "insult" to the tip of a dividing bronchus (shown in the centre of the diagram), each satellite sketch represents a possible pathological consequence at the next stage of development. This forms the basis of the eventual lesion. 
chial buds are supplied by a systemic capillary plexus derived from the primitive aorta. This plexus regresses as the growing lung advances, the developing pulmonary artery taking over. ${ }^{2529} 31$

The pathological options that follow an insult to the tip of a developing bronchus can be graphically represented by the spokes of a wheel (fig 5). The "insult" is undetermined and could take the form of localised trauma, ischaemia, infection, adhesions, or any non-specific injury. It is not only the nature of the insult, however, but also-and probably more importantly-the timing and severity that determine the morphology of the eventual lesion. ${ }^{30}$

The first possibility ( 1 in fig 5 ) is that development of the bronchus and its pulmonary artery supply is totally arrested, leading to agenesis of that lung, lobe, lobule, or segment. The timing will determine how distal the lesion is.

A second possibility ( 2 in fig 5 ) is that the insult may be minor, resulting in a localised abnormality followed by normal development of the distal bronchial tree, lung parenchyma, and pulmonary artery. This will result in a local lesion, such as bronchial stenosis or a bronchogenic cyst.

A third possibility ( 3 in fig 5 ) is that there is no interference with continued development of the bronchial tree, but pulmonary artery growth is arrested. Devoid of pulmonary blood supply, continued growth of the developing bronchial branch could be supported only by vessels retained from its normally regressing systemic capillary network. Growing along with the developing bronchial tree, these systemic vessels would become substantial channels, single or multiple, and establish a systemic arterial supply to the area of lung concerned. The sites of origin of these vessels move distally with the caudal "shift" of the growing primitive aorta from which they arise, ending up in the lower thorax or upper abdomen. Such an anomalous area of "normal" lung with systemic artery supply has been very well shown in a $41 \mathrm{~mm}$ embryo by Boyden. ${ }^{25}$ These aberrant arteries are distinct from bronchial arteries, which are found to occur normally in these patients. ${ }^{25}$

In most instances, in an area of lung with an abnormal arterial blood supply the tracheobronchial tree will be affected to some degree, so that there will be overlap with the following category. ${ }^{32} 33$

In a fourth possibility (4 in fig 5), the insult is severe enough to disrupt both airway and pulmonary arterial development so that a systemic arterial supply is retained to the subsequent abnormal area of lung parenchyma. This is a combined bronchoarterial pulmonary malinosculation, and in its purest form would comply with most descriptions of "classical" sequestration.

The final possibility (5 in fig 5 ) is that, although there is considerable or complete disruption of th $\overrightarrow{\bar{F}}$ tracheobronchial connection, the pulmonary artery continues to develop and supply the abnormal lun segment. This will result in tracheopulmonary $\overline{\overline{\bar{w}}}$ bronchopulmonary malinosculation with normal puf monary artery blood supply, and include such entities as congenital lung cysts, so called sequestration with normal pulmonary artery supply, and cystic ade nomatoid malformation. Peripheral lesions of termit nal and respiratory bronchioles and alveoli (fow example, congenital lobar emphysema) suggest $\stackrel{\overrightarrow{\mathrm{a}}}{\mathrm{a}}$ defect occurring late in development. Congenită lobar emphysema with systemic arterial blood supplit has been described, supporting its embryologicap relationship to other forms of malinosculation. 옹

This leaves extrapulmonary lung anomalies. In the early stages the developing lung bud, with its surrounding mesenchyme, lies in close proximity to the primitive foregut, from which it is derived. ${ }^{24}{ }^{28} \mathrm{An} \overline{\mathrm{W}}$ insult or adhesion in an area where these two developing organs are adjacent is therefore likely to affece both structures; again, the timing and severity of the insult is important to the eventual outcome. NoB mally, the lung develops along genetically determinee lines, the dividing epithelial cells being influenced bo humoral factors from the surrounding meser chyme. $^{1034}$ Differentiating lung epithelial cells drawn by adhesions towards the primitive foregu $\overrightarrow{\vec{G}}$ may come to lie in mesenchyme, which is not condu cive to their normal development. This would resulf in dysmorphogenesis of the misplaced tissue. ${ }^{10 \frac{34}{3}}$ Primitive communications between this ectopic tissure and adjacent organ may develop at any stage. ${ }^{10} 26$ Subsequent development of the pleura could sequess trate this tissue from its parent organ, either partially or completely, resulting in extrapulmonary seques tration or, alternatively, ectopic foregut derivatives within the lung. This hypothesis supports the contentions of ourselves and others 3681527 that intra? pulmonary and extrapulmonary lesions have a similat origin, without the need for an "accessory lung bud플. theory. ${ }^{28}$ It also explains the high incidence of assoc 5 ated congenital foregut anomalies occurring witb extrapulmonary lung anomalies.

The development of the venous drainage is less wetp understood; but when anomalies of venous drainage occur in association with pulmonary malinosculation they are likely to be secondary to the primary lesiow and must be incorporated in the classification. Primary congenital anomalous venous drainage and anomalous systemic pulmonary artery collaterals se ondary to congenital heart disease are not associated with any primary lung problem ${ }^{35} 36$ and are therefore not included in the pulmonary malinosculation spe? trum.

An interesting point is the absence of seconda 
parenchymal changes in patients with pulmonary systemic collaterals secondary to congenital heart disease (for example, in pulmonary atresia) even after many years. This confirms that the secondary changes that occur in malinosculation syndromes are not due to systemic pressures alone, but that there must be some underlying, inherent connective tissue abnormality within the lung neonatally. ${ }^{31}$ This is contrary to earlier hypotheses that parenchymal changes in malinosculation syndromes were primarily a systemic pressure effect. ${ }^{24}$

\section{Summary}

The pulmonary sequestration spectrum and related congenital lung anomalies present an extremely complex and varied group of bronchopulmonary-vascular malformations. Previous attempts at nomenclature and classification have proved inadequate. In this article we present a classification of the newly named pulmonary malinosculation spectrum, which includes all congenital lung anomalies where there is abnormal connection (that is, malinosculation) of one or more of the four major components of lung tissuenamely, tracheobronchial airway, lung parenchyma, arterial supply, and venous drainage, which in various combinations make up these lesions.

We feel that this simple descriptive approach will improve our understanding and management of these complicated lesions and this is supported by the clinical experience we report in the next article.

We would like to thank Professor Brian Corrin, Department of Pathology, for his helpful advice and criticism.

\section{References}

1 Pryce DM. Lower accessory pulmonary artery with intralobular sequestration of lung: a report of seven cases. J Pathol 1946;58:457-67.

2 Pryce DM, Holmes Sellors T, Blair LG. Intralobar sequestration of lung associated with an abnormal pulmonary artery. Br J Surg 1947;35:18-29.

3 Carter R. Pulmonary sequestration. Ann Thorac Surg 1969;7:68-83.

4 Choplin RH, Siegel MJ. Pulmonary sequestration: six unusual presentations. Am J Radiol 1980;134:695-700.

5 Flisak ME, Chandrasekar AJ, Marsan RE, Ali MM. Systemic arterilisation of lung without sequestration. Am J Radiol 1982;138:751-3.

6 Haller JA, Golladay ES, Pickard LR, Tepas JJ, Shorter NA, Shermeta DW. Surgical management of lung bud anomalies: lobar emphysema, bronchogenic cyst, cystic adenomatoid malformation, and intralobar pulmonary sequestration. Ann Thorac Surg 1979;28: 33-43.
7 Chiu RCJ, Herba MJ, Voloria J, Mulder DS. Thoracopulmonary hypogenesis with systemic arterypulmonary vessel fistulae: report of a case. Ann Thorac Surg 1981;31:360-3.

8 Culiner MM. Intralobar bronchial cystic disease, the "sequestration complex" and cystic bronchiectasis. Dis Chest 1968;53:462-9.

9 Takahashi M, Ohno M, Mihara K, Matsuura K, Sumiyoshi A. Intralobar pulmonary sequestration. Radiology 1975;114:543-9.

10 Sade RM, Clouse M, Ellis FH. The spectrum of pulmonary sequestration. Ann Thorac Surg 1974;18: 644-55.

11 Derksen OS. Scimitar syndrome and pulmonary sequestration. Radiol Clin 1977;46:81-93.

12 Thilenius OG, Ruschhaupt DG, Replogle RL, Bharati S, Herman T, Arcilla RA. Spectrum of pulmonary sequestration: association with anomalous pulmonary venous drainage in infants. Pediat Cardiol 1983;4:97-103.

13 Heitzman ER. The lung: radio-pathologic correlations anomalies of pulmonary parenchymal and peripheral vascular development: the sequestration spectrum. 2nd ed. Philadelphia: CV Mosby, 1984:17-38.

14 Macpherson RI, Whytehead L. Pseudosequestration. J Can Assoc Radiol 1977;28:17-25.

15 Golding MR, Kwon K, Chiu CJ, Nicastri AD. Pulmonary sequestration. $J$ Thorac Cardiocasc Surg 1967;54:121-5.

16 Alivizatos P, Cheatle T, de Leval M, Stark J. Pulmonary sequestration complicated by anomalies of pulmonary venous return. J Pediat Surg 1985;20:76-9.

17 Kirks DR, Kang PE, Free EA, et al. Systemic arterial supply to normal basilar segments of the left lower lobe. Am J Roentgenol 1976;126:817-21.

18 Neill CA, Ferencz C, Sabiston DC, Sheldon H. The familial occurrence of hypoplastic right lung with systemic arterial supply and venous drainage "scimitar syndrome". Bull John Hopkins Hosp 1960;107:1-21.

19 Folger GM. The scimitar syndrome: physiologic, development and therapeutic considerations. Angiology 1976;27:373-407.

20 Kuiper-Oosterwal $\mathrm{CH}$, Moulaert A. The scimitar syndrome in infancy and childhood. Eur $J$ Cardiol 1973;1:55-61.

21 Buntain WL, Isaacs H, Payne VC, Lindesmith GG, Rosenkrantz JG. Lobar emphysema, cystic adenomaloid malformation, pulmonary sequestration, and bronchogenic cyst in infancy and childhood: a clinical group. J Pediat Surg 1974;9:85-93.

22 Demos NJ, Teresi A. Congenital lung malformations: a unified concept. J Thorac Cardiovasc Surg 1975;70:260-4.

23 Biancalana L. Die Lungenzysten. Thorax-Chirurge 1963;9:511-31.

24 Abbey Smith R. A theory of the origin of intralobar sequestration of lung. Thorax 1956;11:10-24.

25 Boyden EA. Bronchogenic cysts and the theory of intralobar sequestration: new embryologic data. $J$ Thorac Surg 1958;35:604-15.

26 Gerle RD, Jaretzki A, Ashley CA, Berne AS. Congenital bronchopulmonary-foregut malformation. $N$ Engl J 
Med 1968;278:1413-7.

27 Iwai $\mathrm{K}$, Shindo G, Hajikano H, et al. Intralobar pulmonary sequestration, with special reference to developmental pathology. Am Rev Respir Dis 1973;107:911-20.

28 Eppinger H, Schauenstein W. Krakbeitene der Lungen. Erg Allg Pathol 1902;8:267.

29 Reid LM. Lung growth in health and disease. $\mathrm{Br} \mathrm{J}$ Dis Chest 1984;78:113-34.

30 Stovin PGI. Early lung development. Thorax 1985;40:401-4.

31 Gerbeaux J. Paediatric respiratory disease. 3rd ed. London: Wiley, 1985:185.

32 Blesovsky A. Pulmonary sequestration. Thorax
1967;22:351-7.

33 Shimuzi K. Pulmonary sequestration, with special refe ence to pathogenesis of polycystic changes. Trans $S \overline{\sigma g}$ Pathol Jap 1969;58:180-6.

34 Dameron F. The influence of various mesenchyma om the differentiation of the pulmonary epithelium of the chick embryo in culture in vitro. $J$ Embryol Exp Mo phol 1961;9:268-74.

35 Neill CA. Development of pulmonary veins with refe $\overrightarrow{0}$ ence to the embryology of anomalous venous returi. Pediatrics 1956;18:880-8.

36 Edwards JE. Pathologic and development considerations in anomalous pulmonary venous return. Mayo Clin 离 Proc 1953;28:441-8. 